\title{
Metabonomic and Microbiological Analysis of the Dynamic Effect of Vancomycin-Induced Gut Microbiota Modification in the Mouse
}

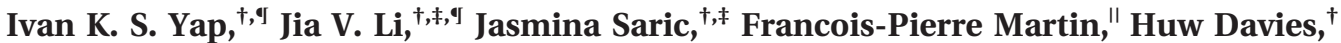 \\ Yulan Wang, ${ }^{\dagger}$ Ian D. Wilson, ${ }^{\$}$ Jeremy K. Nicholson, ${ }^{\dagger}$ Jürg Utzinger, $^{\ddagger}$ Julian R. Marchesi, $^{\perp, \#}$ and \\ Elaine Holmes*, \\ Department of Biomolecular Medicine, Division of Surgery, Oncology, Reproductive Biology and Anaesthetics, \\ Faculty of Medicine, Imperial College London, Sir Alexander Fleming Building, South Kensington Campus, \\ London SW7 2AZ, United Kingdom, Department of Public Health and Epidemiology, Swiss Tropical Institute, \\ P.O. Box, CH-4002 Basel, Switzerland, Nestlé Research Center, P.O. Box 44, Vers-chez-les-Blanc, \\ CH-1000 Lausanne 26, Switzerland, Department of Drug Metabolism and Pharmacokinetics, AstraZeneca, \\ Mereside, Macclesfield, Cheshire SK10 4TG, United Kingdom, Alimentary Pharmabiotic Centre and Department \\ of Microbiology, University College Cork, Western Road, Cork, Ireland, and School of Biosciences, Cardiff \\ University, Cardiff CF10 3TL, Wales, United Kingdom
}

Received December 20, 2007

\begin{abstract}
The effects of the antibiotic vancomycin $(2 \times 100 \mathrm{mg} / \mathrm{kg} / \mathrm{day})$ on the gut microbiota of female mice (outbred NMRI strain) were studied, in order to assess the relative contribution of the gut microbiome to host metabolism. The host's metabolic phenotype was characterized using ${ }^{1} \mathrm{H}$ NMR spectroscopy of urine and fecal extract samples. Time-course changes in the gut microbiotal community after administration of vancomycin were monitored using 16S rRNA gene PCR and denaturing gradient gel electrophoresis (PCR-DGGE) analysis and showed a strong effect on several species, mostly within the Firmicutes. Vancomycin treatment was associated with fecal excretion of uracil, amino acids and short chain fatty acids (SCFAs), highlighting the contribution of the gut microbiota to the production and metabolism of these dietary compounds. Clear differences in gut microbial communities between control and antibiotic-treated mice were observed in the current study. Reduced urinary excretion of gut microbial co-metabolites phenylacetylglycine and hippurate was also observed. Regression of urinary hippurate and phenylacetylglycine concentrations against the fecal metabolite profile showed a strong association between these urinary metabolites and a wide range of fecal metabolites, including amino acids and SCFAs. Fecal choline was inversely correlated with urinary hippurate. Metabolic profiling, coupled with the metagenomic study of this antibiotic model, illustrates the close inter-relationship between the host and microbial "metabotypes", and will provide a basis for further experiments probing the understanding of the microbial-mammalian metabolic axis.
\end{abstract}

Keywords: Butyrate $\bullet$ Co-metabolism • Feces • Metabolomics • Metabonomics • Microbiota • Mouse • NMR • Pattern Recognition • Propionate • Urine • Vancomycin

\section{Introduction}

The mammalian gut contains hundreds of species of commensal and symbiotic microbes, which mainly reside in the large intestine, and the cecum for rodents, where they contribute to $\sim 60 \%$ of the total fecal mass produced. ${ }^{1,2}$ It is widely acknowledged that the presence of the gut microbiota is a key factor in gut development, structure and function. ${ }^{3-5}$ Intestinal microbiota may also play a major role in the etiology of many

* Correspondence should be addressed to Prof. E. Holmes (e-mail: elaine.holmes@imperial.ac.uk). Tel: +44 (0)20 75943220.

${ }^{\dagger}$ Imperial College London.

ฯ These authors contributed equally to this work.

‡ Swiss Tropical Institute.

"Nestlé Research Center.

$\S$ AstraZeneca.

${ }^{\perp}$ University College Cork.

* Cardiff University. gut disorders, such as irritable bowel disease (IBD) and colon cancer. ${ }^{6,7}$ Moreover, the microbiome has recently been reported to vary significantly between obese individuals (human and animals) and normal individuals, and it is known to provide refined control mechanisms on energy recovery through catabolism of otherwise poorly digestible nutrients, that is, resistant starch and other polysaccharides. ${ }^{8-10}$ Since the microbiota are extremely metabolically active and co-metabolize many endogenous and xenobiotic compounds, they clearly have the potential to affect the biochemical composition of the tissues and biofluids of the host. ${ }^{11,12}$

Previous research revealed the influence of the gut microbiota on the urinary metabolite composition in rodent studies using metabolic profiling methods based on ${ }^{1} \mathrm{H}$ nuclear magnetic resonance (NMR) spectroscopy. ${ }^{11-17}$ In particular, diet, medication and other environmental-induced effects on aro- 
matic components such as hippurate and 3-hydroxyphenylpropionic acid (HPPA), which are known to be generated or co-metabolized by gut microbiota, are well-documented in conjunction with a variety of other metabolic pathways such as those involved with bile acid catabolism, choline metabolism and utilization of short chain fatty acids (SCFAs). ${ }^{11-17}$ Further evidence of the direct contribution of the microbiota to host metabolism has been obtained using metabolic profiling methods to characterize germ-free and antibiotic-rodent models. $^{12,13,18,19}$

Although germ-free models provide valuable information on microbial-mammalian co-metabolism, acting in some respects as a "knock out", it could be argued that, since the gut of such animals remains underdeveloped, they do not offer a true picture of 'normal' mammalian physiology and as such are not an optimal model for characterizing microbiota-mammalian metabolic interaction in 'normal' animals. An alternative approach is to employ conventional animals, with normal gut physiology, combined with antimicrobial treatment, which mimics more closely the actual environment of the host gut and its commensals. Indeed, from studies aimed at the deliberate reduction of the gut microbiota, as well as on the investigation of antibiotic-induced nephrotoxicity of pharmacological effects on the gut microbiota, a reduction in the urinary excretion of hippurate and other phenolic metabolites was evident. $^{13,20,21}$

As part of ongoing studies to determine the effect of the gut microbiota on host metabolic phenotypes, we employ here an antibiotic-based model to directly probe the dynamic effect of the microbiotal contribution to urinary and fecal composition. Additionally, we monitor time-course changes in the gut microbiota community during the course of the recovery using 16S rRNA gene polymerase chain reaction (PCR) and denaturing gradient gel electrophoresis (DGGE) analysis. For this, we have used vancomycin (a glycopeptide antibiotic, ${ }^{22}$ with broad activity against Gram-positive bacteria), which causes marked decrease in gut microbiotal populations in mice. ${ }^{23}$ Vancomycin was chosen as a means of reducing the Gram-positive bacteria in the gut without overtly modifying the Gram-negative microbes, in order to target one particular aspect of the microbial influence on host metabolism. Additionally, vancomycin is known to be poorly partitioned across the mammalian gastrointestinal mucosa, and since it does not have systemic absorption, should not perturb host biochemistry directly. ${ }^{23}$

\section{Methods}

Two studies were initiated to assess effects of antibiotic treatments on host-gut metabolism. The initial exploratory study was used to monitor metabolic changes in the mouse at a single time point. A second study, hereafter referred to as the extension study, was carried out to ascertain the dynamic metabolic effects of antibiotic treatments in mice.

Exploratory Study Design. This study was carried out at the animal care facilities of the Swiss Tropical Institute (Basel, Switzerland), and complied with Swiss local and national regulations on animal welfare (permission no. 2081). Twelve female (age, $\sim 3$ weeks; weight, 20-25 g) outbred NMRI strain mice were purchased from RCC (Füllinsdorf, Switzerland). The animals were kept in groups of 3 in macrolon cages, and acclimatized for 7 days prior to the study under controlled environmental conditions (temperature, $\sim 22{ }^{\circ} \mathrm{C}$; relative humidity, $60-70 \%$; day/night cycle, $12-12 \mathrm{~h}$ ). Mice were fed commercial rodent feed with water ad libitum. Six of the mice were dosed with vancomycin hydrochloride $(2 \times 100 \mathrm{mg} / \mathrm{kg} /$ day) on day 0 of the study for 2 successive days. Mice were treated orally by gastric intubation with vancomycin suspended in $7 \%$ Tween-80 and $3 \%$ ethanol (volume $10 \mathrm{~mL} / \mathrm{kg}$ body weight; serving as vehicle). The remaining six mice were given the vehicle only (10 $\mathrm{mL} / \mathrm{kg}$ body weight), and hence served as controls.

Fecal and urine sample collection was carried out 2 days after the initial dosing (i.e., day 2) between 09.00 and $11.00 \mathrm{~h}$. From each mouse, urine (at least $20 \mu \mathrm{L}$ ) and fecal samples (1-2 pellets) were collected into Petri dishes by gently stretching the mice or rubbing their abdomen. Samples were transferred into small microcentrifuge tubes, immediately frozen over dry ice and stored at $-80^{\circ} \mathrm{C}$.

Extension Time-Course Study Design. A total of 20 NMRI female mice were purchased from RCC and divided into the control and vancomycin-treated groups with 10 mice each. Each mouse from the vancomycin treatment group was treated in the same way as the first study at 15-week-old, while mice from the control group were administered 3 doses of drug vehicle only (age: 14 weeks). The 1-week difference in the age at the start of the experiment between this and the exploratory study can be ignored since mice are fully mature at this age and thus their microbial community would be fully established. Urine and stool samples for NMR experiments were collected from both groups at 1 day predosing and days $1,2,3,5,7,13$, and 19 after the final dosing and stored at $-40{ }^{\circ} \mathrm{C}$. Stool samples for microbiological investigation were also collected at the same time points, fixed in $0.5 \mathrm{~mL}$ of $4 \%$ formalin, to which $9.5 \mathrm{~mL}$ of $70 \%(\mathrm{v} / \mathrm{v})$ ethanol was added and stored at $-20{ }^{\circ} \mathrm{C}$.

Sample Preparation. Urine samples were prepared by mixing $20 \mu \mathrm{L}$ of urine with $30 \mu \mathrm{L}$ of a phosphate buffer containing $90 \% \mathrm{D}_{2} \mathrm{O}$ and $0.25 \mathrm{mM}$ 3-trimethylsilyl-1-[2,2,3,3${ }^{2} \mathrm{H} 4$ ] propionate (TSP) and left to stand for $10 \mathrm{~min}$. The resulting mixtures were then transferred into $1.7 \mathrm{~mm}$ (outer diameter) NMR capillary tubes.

Each fecal sample was homogenized with buffer ( $1 \mathrm{~mL}, 0.2$ $\mathrm{M}$ sodium phosphate, $\mathrm{pH}$ 7.4). Homogenates were sonicated at ambient temperature $\left(25^{\circ} \mathrm{C}\right)$ for $30 \mathrm{~min}$ and centrifuged at $11000 \mathrm{~g}$ for $10 \mathrm{~min}$. The supernatants $(460 \mu \mathrm{L})$ were aliquoted and a final volume of $700 \mu \mathrm{L}$ was made by the addition of 240 $\mu \mathrm{L}$ of TSP in $90 \% \mathrm{D}_{2} \mathrm{O}$. These were further centrifuged at $11000 \mathrm{~g}$ for $10 \mathrm{~min}$ and $600 \mu \mathrm{L}$ of the supernatant was pipetted into 5 mm (outer diameter) NMR tubes.

${ }^{1}$ H NMR Spectroscopy. Spectra were obtained on a Bruker DRX 600 spectrometer (Bruker Biospin; Rheinstetten, Germany) at $600.13 \mathrm{MHz}$ (ambient probe temperature $27^{\circ} \mathrm{C}$ ). A standard 1-dimensional (1D) pulse sequence was used [recycle delay (RD) $-90^{\circ}-t_{1}-90^{\circ}-t_{\mathrm{m}}-90^{\circ}$-acquire free induction decay (FID)]. The water signal was suppressed by irradiation during RD of $2 \mathrm{~s}$, and mixing time $\left(t_{\mathrm{m}}\right)$ of $150 \mathrm{~ms}$. $t_{1}$ was set to $3 \mu$ s and the $90^{\circ}$ pulse length was adjusted to $\sim 10 \mu$ s. For each sample, a total of 64 transients (in exploratory study) and 256 transients (in the extension study) were accumulated into $\sim 32000$ data points using a spectral width of $20 \mathrm{ppm}$. Prior to Fourier transformation, all FIDs were multiplied by an exponential function equivalent to a line broadening of $0.3 \mathrm{~Hz}$. The assignment of the peaks to specific metabolites was based on 2-dimensional (2D) ${ }^{1} \mathrm{H}-{ }^{1} \mathrm{H}$ correlation spectroscopy (COSY) and ${ }^{1} \mathrm{H}-{ }^{1} \mathrm{H}$ total correlation spectroscopy (TOCSY) NMR, published literature, ${ }^{24,25}$ and statistical total correlation spectroscopy (STOCSY). ${ }^{26}$ 
Data Processing and Analysis. ${ }^{1} \mathrm{H}$ NMR spectra of fecal extract and urine samples were manually phased and baseline corrected using XwinNMR 3.5 (Bruker Biospin; Rheinstetten, Germany). The ${ }^{1} \mathrm{H}$ NMR spectra were referenced to the TSP resonance at $\delta 0.0$. The spectra were digitized using a MATLAB (version 7, The Mathworks, Inc.; Natwick, MA) script developed in-house. The region containing the water resonance was removed from each spectrum to eliminate baseline effects of imperfect water saturation. For each spectrum, normalization to the total sum of the residual spectrum was carried out prior to pattern recognition analyses followed by scaling of the data to unit variance.

Principal component analysis (PCA) was applied to the processed spectral data to reveal intrinsic treatment-related patterns within data, as well as monitoring the dynamic response of the mice to vancomycin intervention. Interanimal variation can confound data interpretation, particularly in multivariate data of high dimensionality. Therefore, orthogonalprojection to latent structure discriminant analysis (O-PLSDA) ${ }^{27}$ was performed in a MATLAB environment to optimally model class differences and to systematically identify metabolites contributing to the differences between the vancomycintreated and control groups. The O-PLS-DA method decomposes the variation in $X$ (NMR data) into 3 parts; the first being the variation in $X$ related to $Y$ (the class variable), and the last 2 containing the specific systemic variation in $X$ and residual, respectively. The contribution of each metabolite to sample classification is interpreted using the O-PLS coefficients with back-scaling transformation, indicating the variable contributions to the antibiotic-induced discrimination in the models. ${ }^{28}$ Here, the colors projected onto the spectrum indicate the correlation of the metabolites discriminating vancomycintreated mice from the corresponding controls. Red indicates a high correlation and dark blue denotes no correlation with sample class. The direction and magnitude of the signals relate to the covariation of the metabolites with the classes in the model. A coefficient of 0.71 , corresponding to $5 \%$ significance level, was used as a cutoff value to select variables that had a significant correlation with class and the model predictive performance (robustness) was evaluated using a 7 -fold cross validation method. ${ }^{28}$

Two of the 3 urinary metabolites that were most highly correlated with antibiotic ingestion, namely, hippurate and phenylacetylglycine, were regressed against the total fecal metabolite profiles using O-PLS analysis based on the integrated hippurate resonance at $\delta^{1} 7.84 \mathrm{ppm}$, or the integrated phenylacetylglycine resonance at $\delta^{1} 7.43 \mathrm{ppm}$, as the $Y$ (response) matrix. The third metabolite, guanidinoacetate, was not integrated due to substantial peak overlap, which would lead to inaccuracy in the calculation.

Microbiological Analysis (PCR-DGGE Experiment) and DNA Extraction. Approximately $0.2 \mathrm{~g}$ of stool sample was processed to remove preservation solution (2 washes with 0.5 $\mathrm{mL}$ of PBS) and mixed with $1.4 \mathrm{~mL}$ of buffer ASL; the suspension was transferred into a plastic tube containing $1 \mathrm{~g}$ of $0.1 \mathrm{~mm}$ diameter zirconium/glass beads and vortexed for 1 min at maximum speed to ensure the sample was thoroughly homogenized. The DNA extraction was carried out using QIAamp DNA Stool Mini Kit (Qiagen, Dublin, Ireland) according to the manufacturer's instructions. The $16 \mathrm{~S}$ rRNA gene was amplified using a nested approach; the first PCR used primers 27f (5’ GAGTTTGATCMTGGCTCAG 3') and 1492r (5' GGYTACCTTGTTACGACTT 3') and the second PCR was performed with
341fGC primers (5' CGCCCGCCGCGCGCGGCGGGCGGGGCGGGGGCACGGGGGTACG-GGAGGCAGCAG 3') and 519r primers (5' GTATTACCGCGGCTGGCTG 3'). DGGE analysis was performed as described elsewhere. ${ }^{30}$ A number of strong discriminatory DGGE bands were excised with a sterile pipet tip into sterile water, amplified and cleaned (QIAquick PCR Purification Kit, Qiagen, Dublin, Ireland) for the sequencing. The amplicon was purified (QIAquick PCR Purification Kit, Dublin, Ireland) and sequenced by GATC (Konstanz, Germany), phylogenetic analysis was performed using the RDP classifier tool. ${ }^{31}$ Additional PCR was performed using specific primers (Clostridium leptum subgroup or the Clostridium coccoides subgroup) based on the extracted DNA and the PCR products were visualized on agarose gels by staining with ethidium bromide. $^{31}$

\section{Results}

Data obtained from both the exploratory and extension studies revealed consistent changes in the urinary and fecal metabolic profiles with the time course study generating further information on recovery of individual metabolites and the global profile after antibiotic intervention. The control and treated groups excreted drug vehicle (7\% Tween-80 and 3\% ethanol) in their feces on day 1 but this was totally eliminated within $48 \mathrm{~h}$. No significant endogenous changes in metabolite profile were observed in the first 3 principal components (PCs).

Vancomycin-Induced Effects on the ${ }^{\mathbf{1}} \mathrm{H}$ NMR Spectroscopic Profiles of Urine Samples. Typical examples of the ${ }^{1} \mathrm{H}$ NMR spectra of urine obtained from an untreated and a vancomycintreated mouse are shown in Figures 1A and 1B. Spectra from control mice were dominated by a number of metabolites, namely, $\alpha$-ketoglutarate, $\alpha$-ketoisocaproate, $\alpha$-ketoisovalerate, $n$-butyrate, citrate, creatine, creatinine, dimethylamine, fumarate, glycine, hippurate, lactate, $N$-acetyl glycoprotein fragments, phenylacetylglycine, succinate, taurine, trimethylamine, and trimethylamine- $N$-oxide. Visual inspection of the spectra revealed marked differences in overall metabolic composition between urines obtained from untreated and vancomycintreated mice, with the latter showing relatively lower concentrations of urinary phenylacetylglycine, hippurate and fumarate, and higher levels of urinary creatine, $\alpha$-ketoisocaproate and $\alpha$-ketoisovalerate. To examine the metabolic disturbance resulting from vancomycin in urine over all the time points, a PCA strategy was applied to the entire data set. A total of 3 PCs were calculated for a PCA model using unit variance scaled spectral data shown in Figure 1C. There was clear separation between urine samples obtained from control mice and mice given vancomycin 1-3 days after the final dosing. At days 5 and 7 post-treatment, samples from treated mice were still differentiated but moved closer to the control cluster, finally co-mapping with the control cluster by day 13 post-treatment.

In addition to visually identified metabolites, O-PLS-DA (Figure 2) highlighted further discriminatory metabolites for the antibiotic-treated group, with vancomycin-treated mice showing lower levels of urinary trimethylamine and trimethylamine- $N$-oxide, and the higher levels of urinary $n$-butyrate, guanidinoacetic acid and $N$-acetyl glycoprotein fragments. However, Student's $t$-tests of the urinary NMR data revealed that only guanidinoacetic acid and hippurate showed statistical significance at a 5\% significance level. The model parameters for urine samples are given in Table 1 together with the O-PLS- 


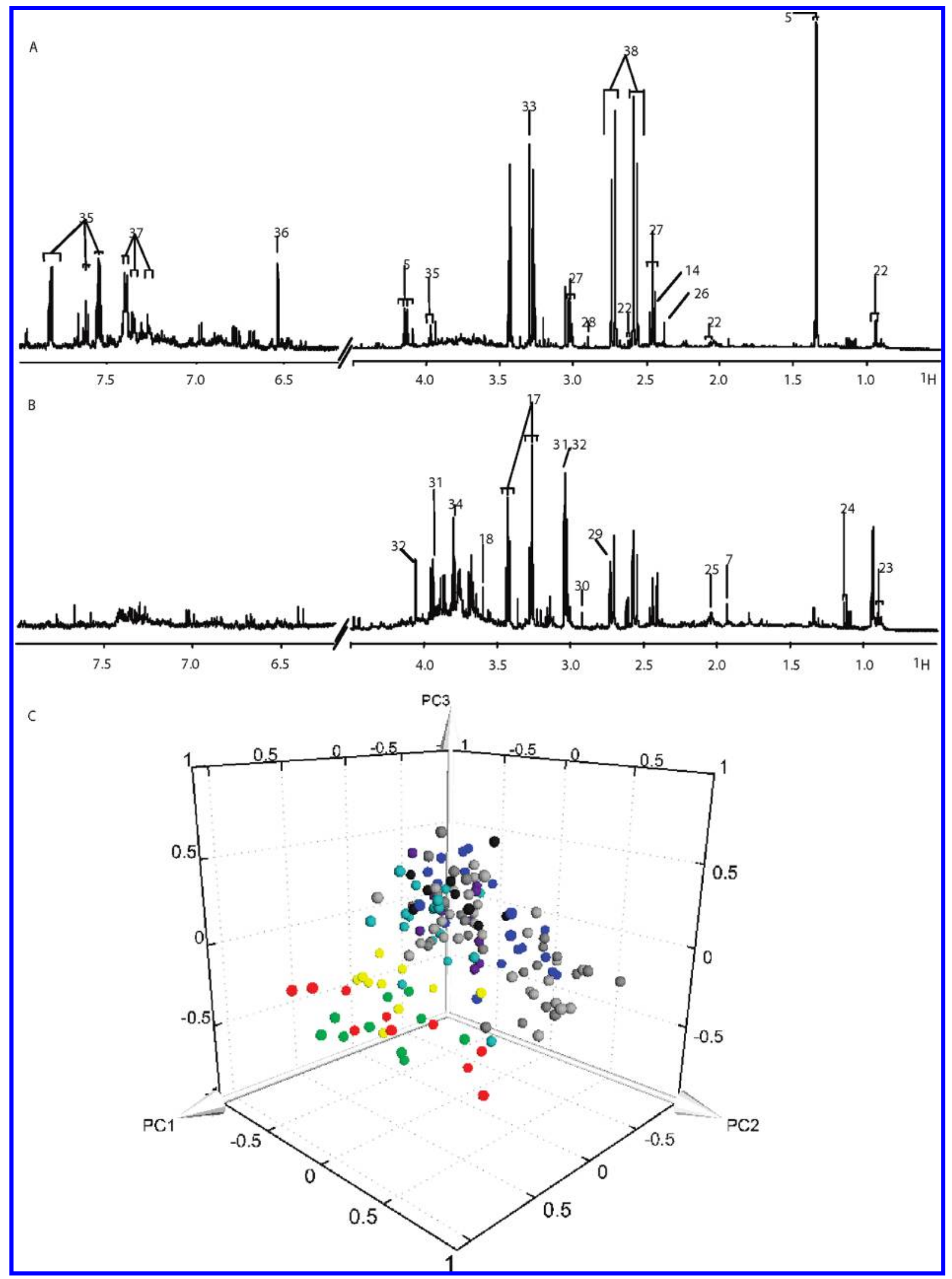

Figure 1. Typical $600 \mathrm{MHz}{ }^{1} \mathrm{H}$ NMR spectra of urine obtained from untreated mice (A) and vancomycin-treated mice (B) 2 days after the final dosing. (C) PCA trajectory plots of urine. Color code: gray (control), black (vancomycin-treated d-1), red (d1), green (d2), yellow (d3), blue (d5), purple (d7), dark blue (d13 and d19). Key: 5, lactate; 7, acetate; 14, succinate; 17, taurine; 18, glycine; 22, $\alpha$-ketoisocaproate; 23, butyrate; 24, $\alpha$-ketoisovalerate; 25, $\mathrm{N}$-acetyl glycoproteins (NAG); 26, pyruvate; 27, $\alpha$-ketoglutarate; 28, dimethylglycine; 29, dimethylamine; 30, trimethylamine; 31, creatine; 32, creatinine; 33, trimethylamine- $N$-oxide (TMAO); 34 , guanidoacetic acid (GAA); 35 , hippurate; 36 , fumarate; 37 , phenylacetylglycine; 38 , citrate. 


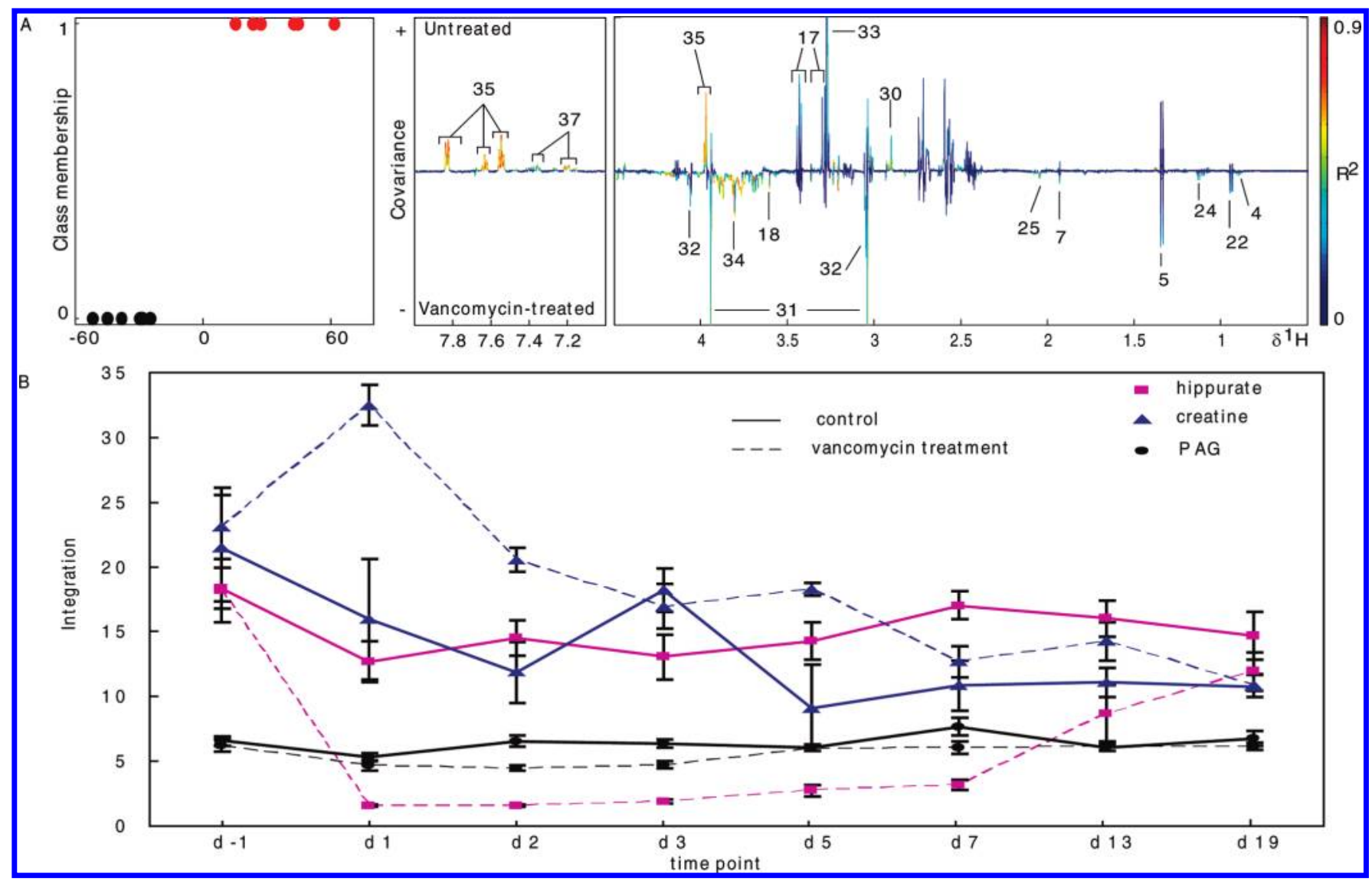

Figure 2. (A) O-PLS-DA scores and coefficient plots derived from the ${ }^{1} \mathrm{H}$ NMR spectra of urine indicating discrimination between untreated and vancomycin-treated mice 2 days after the final dosing. The color code corresponds to the correlation coefficients of the variables. For metabolites identification key, please see Figure 1 caption. (B) Integration of selected peaks from hippurate, creatine and PAG in total area-normalized urinary spectra at all time points, demonstrating the variation in the concentrations of metabolites with the progression of time. Error bars represent the standard error.

DA correlation coefficients indicating the relative contributions of key metabolites contributing to the antibiotic-perturbed profiles.

In urinary O-PLS-DA plots comparing control and treated animals for each of the 7 time points, 3 dominant metabolites, that is, hippurate, phenalactyglycine (PAG) and creatine, were found to co-vary consistently during the course of the recovery from vancomycin treatment. The mean integrated signals for these metabolites relative to the total spectrum were calculated and plotted (Figure 2B). Urinary concentrations of both hippurate and PAG decreased after the vancomycin treatment. However, the "recovery" of hippurate toward control levels was slower (ca. 19 days) than that of PAG (ca. 7 days). Urinary creatine was increased at days 1 and 2 post-vancomycin treatment and recovered from day 3 onward.

Vancomycin-Induced Effects on the Fecal Metabolite Profiles. Typical examples of the ${ }^{1} \mathrm{H}$ NMR spectra of fecal extracts obtained from an untreated and a vancomycin-treated mouse are shown in Figures 3A and 3B. A range of metabolites such as amino acids, SCFAs, uracil and succinate were also directly assigned in spectra from control and vancomycintreated mice consistent with previously published data. ${ }^{16,17,24,25}$ Reduction in the relative concentrations of fecal amino acids, SCFAs and uracil, and an increase in fecal choline and oligosaccharide levels were observed in vancomycin-treated mice. The PCA plot of fecal samples collected over the entire study duration (Figure 3C) showed a similar metabolic move-
Table 1. Metabolic Effects of Vancomycin Treatment on Urine of Mice

\begin{tabular}{|c|c|c|}
\hline metabolites & chemical shift & $\begin{array}{c}\text { untreated vs vancomycin-treated } \\
\text { (correlation coefficient) } \\
\text { urine } \\
Q^{2}=0.75 \\
R^{2}=0.98\end{array}$ \\
\hline$\alpha$-ketoisovalerate & $1.13(\mathrm{~d})$ & $-0.64^{a}$ \\
\hline$n$-butyrate & $0.90(\mathrm{t})$ & $-0.58^{a}$ \\
\hline creatine & $3.93(\mathrm{~s})$ & $-0.60^{a}$ \\
\hline creatinine & $4.06(\mathrm{~s})$ & $-0.45^{a}$ \\
\hline GAA & $3.80(\mathrm{~s})$ & -0.75 \\
\hline glycine & $3.56(\mathrm{~s})$ & $-0.47^{a}$ \\
\hline hippurate & $7.84(\mathrm{~d})$ & +0.86 \\
\hline NAG & $2.06(\mathrm{~s})$ & $-0.64^{a}$ \\
\hline phenylacetylglycine & $7.43(\mathrm{~m})$ & $+0.67^{a}$ \\
\hline taurine & $3.46(t)$ & $+0.49^{a}$ \\
\hline TMA & $2.89(\mathrm{~s})$ & $+0.55^{a}$ \\
\hline TMAO & $3.27(\mathrm{~s})$ & $+0.50^{a}$ \\
\hline
\end{tabular}

${ }^{a}$ Shows trend but not significant at the level of $P<0.05$; GAA, guanidoacetic acid; NAG, $N$-acetyl glycoproteins; TMA, trimethylamine; TMAO, trimethyl- $N$-oxide; s, singlet; d, doublet; t, triplet; m, multiplets; + , relatively higher in control mice; -, relatively lower in control mice.

ment to that observed for the urinary data. At days 1 and 3 post-vancomycin dosing, the fecal water composition was markedly different from that of controls. In terms of the number of perturbed metabolites, vancomycin showed a greater effect in fecal water than in urine. 


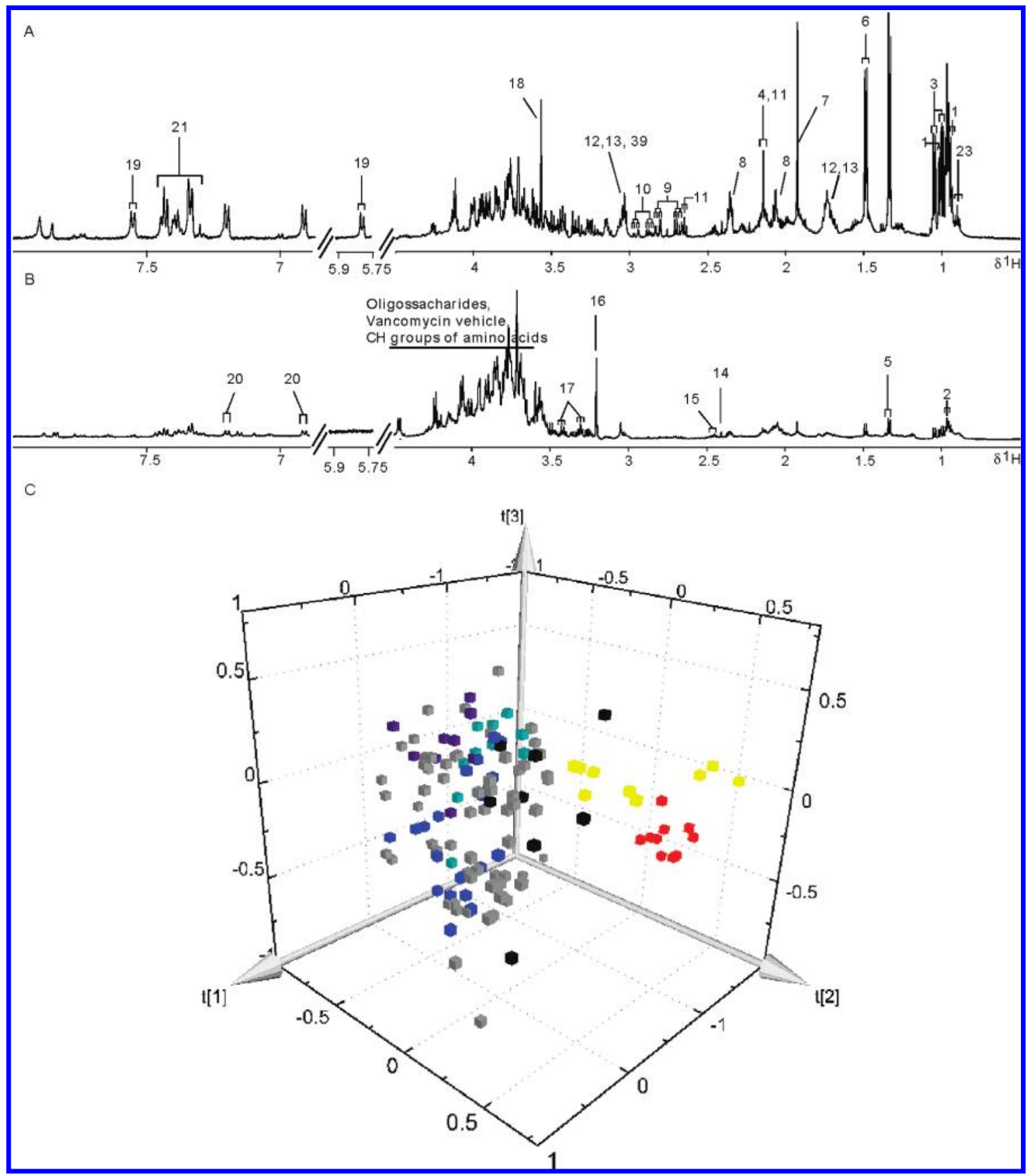

Figure 3. Typical $600 \mathrm{MHz}{ }^{1} \mathrm{H}$ NMR spectra of fecal extracts obtained from untreated mice (A) and vancomycin-treated mice (B) 2 days after the final dosing. (C) PCA trajectory plots of fecal water. Color code: gray (control), black (vancomycin-treated d-1), red (d1), yellow (d3), blue (d5), purple (d7), dark blue (d13 and d19). Keys: 1, isoleucine; 2, leucine; 3, valine; 4, propionate; 5, lactate; 6, alanine; 7, acetate; 8 , glutamate; 9 , aspartate; 10 , asparagine; 11 , methionine; 12 , lysine; 13, arginine; 14 , succinate; 15 , glutamine; 16 , choline; 17 , taurine; 18 , glycine; 19 , uracil; 20 , tyrosine; 21 , phenylalanine; 23 , butyrate; 39,5 -aminovalerate.

Systematic comparison of the treated and control groups using O-PLS generated strong models with goodness of fit, $R^{2}$, of $98.3 \%$ and goodness of prediction, ${ }^{27,28} Q^{2}$, of $92.3 \%$, and showed good discrimination in the scores plot (Figure 2 ), indicating that vancomycin caused a substantial and significant effect on the fecal composition as compared to the urinary changes. The O-PLS coefficients plot (Figure 4) confirmed the observed metabolic changes and also identified decreased levels of fecal lactate and succinate as significant discriminators of vancomycin treatment. The model parameters for fecal samples are given in Table 2, together with the O-PLS-DA correlation coefficients indicat- ing the relative contributions of key metabolites contributing to the antibiotic-perturbed profiles.

The O-PLS-DA coefficient plot derived from spectra data of fecal water at day 1 post-treatment (data not shown) showed a marked depletion of a range of amino acids including leucine, isoleucine, valine, lysine, glutamate, methionine, taurine and aromatic amino acids such as tyrosine, phenylalanine and cytosine, and SCFAs (acetate, butyrate), together with other metabolites, such as 5 -aminovalerate, succinate, nicotinurate and glucose. Moreover, increased concentrations of choline, oligossacharides and fumarate were also observed in the vancomycin-treated mice. All metabolites showed a similar 


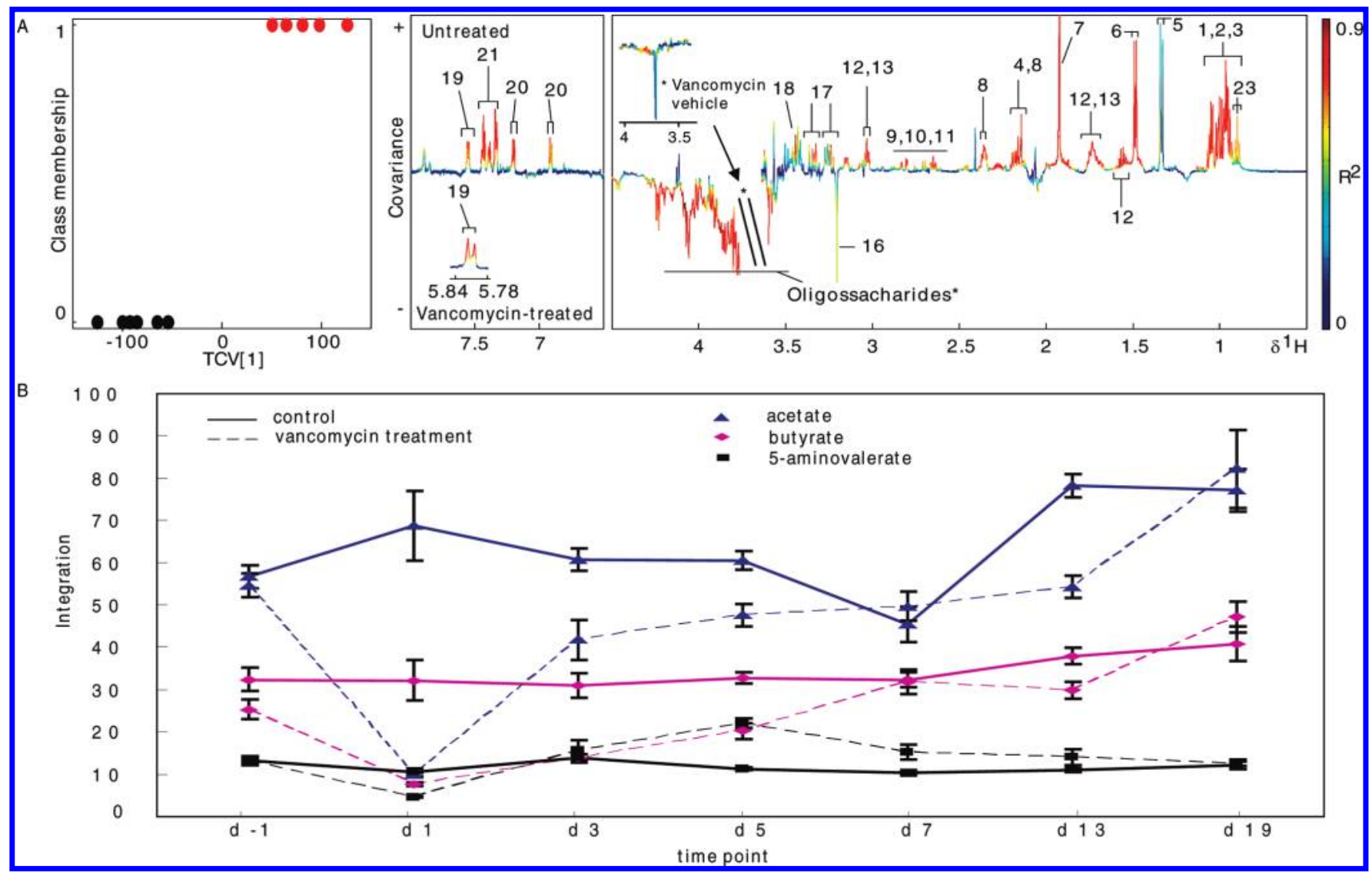

Figure 4. (A) O-PLS-DA scores and coefficient plots derived from the ${ }^{1} \mathrm{H}$ NMR spectra of fecal extract indicating discrimination between untreated and vancomycin-treated mice 2 days after the final dosing. The color code corresponds to the correlation coefficients of the variables. For metabolite identification key, see Figure 3 caption. (B) Integration of selected peaks from acetate, butyrate and 5-aminovalerate in total area-normalized fecal water spectra for all time points, demonstrating the concentrations of metabolites varied as the time progressed. Error bars represent standard error. Key: $\left(^{*}\right)$ Vancomycin vehicle peak did not contribute to the separation of the 2 classes of animal and was therefore removed from the analysis (inset).

trend of changes during the course of treatment except 5-aminovalerate, which fluctuated and recovered back to "normal" level at day 7 post-treatment. The relative concentrations of selected metabolites were calculated based on the integration of peaks, shown in Figure 4B.

O-PLS Correlation of Urinary Hippurate and Phenylacetylglycine Concentrations with Fecal Metabolites. O-PLS regression of urinary hippurate against the fecal extract profiles (Figure 5A) generated a strong model $\left(R^{2}=92.9 \%, Q^{2}=67.8 \%\right.$ using a 1-component model with 1 orthogonal component). Several fecal amino acids were highly correlated with urinary hippurate concentrations, while SCFAs (propionate, $n$-butyrate and acetate) and uracil were also significantly positively correlated with urinary hippurate, albeit to a lesser extent. Conversely, fecal choline and sugars were inversely correlated with urinary hippurate. Lactate and succinate were uncorrelated with hippurate. The regression model for urinary phenylacetylglycine (Figure 5B) showed correlations with the same molecules as hippurate, but the model and correlation coefficients were weaker $\left(R^{2}=86.2 \%, Q^{2}=41.1 \%\right.$ using a 1 -component model with 1 orthogonal component). Subsequent O-PLS regression analyses were carried out on all the time points (day 1-day 19) and the characteristics of these models are summarized in Table 3 . The pattern of changes observed in these models were consistent, with decreasing $Q^{2}$ at later time points, indicating weaker correlations between the fecal metabolic profiles and urinary hippurate and pheny- lacetylglycine concentrations. These findings are consistent with the changes observed in the quantified urinary metabolites (Figure 2B).

Microbial Diversity with Time and Trajectory of DGGE Data. 16S rRNA PCR-DGGE and sequencing, as a routine molecular approach, was applied to examine disturbance of the microbiota caused by vancomycin treatment in mice over the duration of the extension study time course (19 days). Sequences closely related to C. leptum, C. coccoides, Clostridium symbiosum and Photorhabdus luminescens were found to be impacted and were lost from the DGGE profiles 1 day posttreatment (Table 4). However, bands which were shown to be closely related to C. leptum and C. coccoides recovered at day 2 or day 3 post-treatment.

\section{Discussion}

Antibiotics alter the composition of the gut microbiota ${ }^{32}$ and play a central role in the development of Clostridium difficileassociated colitis. ${ }^{33}$ Moreover, the gut microbiota is resilient, and once the antibiotic source has been removed, it is thought to substantially recover to its original composition and diversity. ${ }^{32}$ However, no work to date has shown how a major perturbation of the gut microbiota manifests itself in the host's systemic metabolic phenotype, which is known to be partially under microbiome control. ${ }^{34}$ Metabolites synthesized via microbiome activity influence host biology and any dysbiosis in 
Table 2. Metabolic Effects of Vancomycin Treatment on Feces of Mice

\begin{tabular}{|c|c|c|}
\hline metabolites & $\begin{array}{l}\text { chemical shift of } \\
\text { discriminating } \\
\text { signals }\end{array}$ & $\begin{array}{l}\text { untreated vs vancomycin-treated } \\
\text { (correlation coefficient) } \\
\text { Feces } \\
Q^{2}=0.92 \\
R^{2}=0.98\end{array}$ \\
\hline 5-aminovalerate & $3.02(\mathrm{t})$ & +0.95 \\
\hline acetate & $1.92(\mathrm{~s})$ & +0.88 \\
\hline alanine & $1.48(\mathrm{~d})$ & +0.97 \\
\hline arginine & $1.63(\mathrm{~m})$ & +0.96 \\
\hline asparagine & $2.94(\mathrm{~m})$ & +0.82 \\
\hline aspartate & $2.79(\mathrm{~m})$ & +0.90 \\
\hline$n$-butyrate & $0.90(\mathrm{t})$ & +0.86 \\
\hline choline & $3.23(\mathrm{~s})$ & $-0.69^{a}$ \\
\hline glutamate & $2.08(\mathrm{~m})$ & +0.91 \\
\hline glycine & $3.56(\mathrm{~s})$ & $+0.65={ }^{a}$ \\
\hline isoleucine & $1.02(\mathrm{~d})$ & +0.95 \\
\hline lactate & $1.32(\mathrm{~d})$ & $+0.48^{a}$ \\
\hline leucine & $0.96(\mathrm{t})$ & +0.94 \\
\hline lysine & $1.72(\mathrm{~m})$ & +0.95 \\
\hline methionine & $2.13(\mathrm{~s})$ & +0.93 \\
\hline oligosaccharides & $3.5-4.0$ & -0.90 \\
\hline phenylalanine & $7.40(\mathrm{t})$ & +0.95 \\
\hline propionate & $2.19(\mathrm{q})$ & +0.90 \\
\hline taurine & $3.46(\mathrm{t})$ & +0.81 \\
\hline tyrosine & $6.87(\mathrm{dd})$ & +0.89 \\
\hline uracil & $5.81(\mathrm{~d})$ & +0.91 \\
\hline valine & $1.04(\mathrm{~d})$ & +0.94 \\
\hline
\end{tabular}

a Shows trend but not significant at the level of $P>0.05$; s, singlet; d, doublet; dd, doublet of doublets; $t$, triplet; $m$, multiplets; + , relatively higher in control mice; -, relatively lower in control mice.

this virtual organ has implications for the host health. We undertook to correlate changes in the gut microbiota, induced by exposure to vancomycin, with the host's metabonome ${ }^{35}$ and determine to what extent they are associated.

Results obtained from the exploratory and extension studies revealed consistent changes in the urine and fecal metabolic profiles of mice after vancomycin treatment indicating that the vancomycin-induced effect on the microbiotal community was robust and reproducible. In the extension study, we demonstrated recovery from vancomycin treatment with individual metabolites showing differential recovery rates. We also showed clear differences in gut microbial communities between control and antibiotic-treated mice using PCR-DGGE-based microbiological methods. Since all animals were fed with the same food, kept in the same environment and given drug vehicle, it was unlikely that metabolic variation observed were due to environmental conditions. Neither vancomycin nor its metabolites were detected in any of the urine samples. This was expected since vancomycin does not cross through the intestinal lining, and since feces are excreted within $24 \mathrm{~h}$ after treatment. However, drug vehicle was detected in the fecal profiles on day 1 from both groups and was eliminated within $48 \mathrm{~h}$. Certain drug vehicles have been shown to effect urinary biochemical profiles. However, in a study conducted by Beckwith-Hall et al. $^{36}$ Tween exerted the smallest effect on the metabolic profiles after saline consistent with the present study. ${ }^{36}$ The spectral regions affected by the vehicle were removed from the data set prior to mathematical modeling to minimize its effect on data analyses and interpretation of the metabolic data.

Previous studies have highlighted the reduction in the urinary excretion of hippurate in both germ-free animals ${ }^{12}$ and in rats treated with antibiotics. ${ }^{13}$ After exposure to a standard laboratory environment, a dynamic series of changes in the biochemical composition of the urine was observed in germfree animals, as reflected by sequential excretion of phenylacetylglycine, 4-hydroxyphenylpropionic acid, 3-hydroxyphenylpropionic acid and, finally, hippurate at around 21 days postexposure. Hippurate is a well-described mammalianmicrobial co-metabolite ${ }^{17,37}$ formed by glycine conjugation of benzoate (predominantly in the liver), which is produced from bacterial metabolism of plant phenols, ${ }^{38}$ or by intestinal microbiota in the presence of quinic acid (1,3,4,5-tetrahydroxycyclohexanecarboxylic acid). ${ }^{39}$ Depletion of hippurate has been previously reported after ingestion of antibiotics such as cephaloridine ${ }^{19,20}$ gentamicin, ${ }^{21}$ neomycin, tetracycline hydrochloride and bacitracin mixture. ${ }^{13}$ Gut microbiota also extensively catabolize protein and aromatic amino acids, including phenylalanine, tyrosine and tryptophan. ${ }^{40}$ Fecal amino acids, including tyrosine and phenylalanine, were noted to be directly correlated with urinary hippurate and phenylacetylglycine, consistent with decreased catabolism of these amino acids by the microbiota. In the current study, vancomycin had a strong impact on the region of the spectrum representing phenolic molecules with particular effect on reduction of signals from hippurate and phenylacetylglycine. Both PAG and hippurate were observed to be decreased after administration of vancomycin; however, urinary concentrations of PAG were restored to those of controls at day 5, while recovery of urinary hippurate was not experienced until the end of the experiment at day 19 post-treatment. This suggests that establishment of a stable gut microbiota requires a similar time frame in both conventionalisation of germ-free rats and in conventional mice after vancomycin treatment. Such a 19day recovery of hippurate may reflect a delayed repopulation of hippurate-producing microbiota compared with other microbiota.

Other urinary indicators of vancomycin-perturbed microbiota included relative decrease in the concentrations of trimethylamine (TMA), a gut bacterial product of the choline metabolism, and trimethylamine- $N$-oxide (TMAO), a product of its hepatic detoxification. ${ }^{41,42}$ Choline metabolism is a complex host-symbiont molecular interaction which involves both mammalian and symxenobiotic (metabolite with dietary and/or gut microbiota origin) metabolic pathways. ${ }^{16}$ Although vancomycin treatment did not significantly alter the choline and methylamine content of fecal extracts, a trend towards decreased levels of choline in fecal water from vancomycintreated mice was observed at day 1 postdosing. The variations of TMA and choline concentration were not observed from day 3 onward. This might indicate that choline metabolism is influenced by both vancomycin and nonvancomycin sensitive bacteria. In addition, methylamines are known to be cometabolized by the host and the gut microbiota in the large intestine. $^{42}$

Bacterial fermentation of carbohydrates in the cecum and the large intestine leads to the production of SCFAs and lactic acid depending on the bacterial strains involved. ${ }^{43-46}$ We observed lower amounts of fecal acetate, $n$-butyrate, propionate and lactate, and increased quantities of fecal oligosaccharides in mice treated with vancomycin compared to controls (Table 1), suggesting disruption in carbohydrate fermentation due to vancomycin-induced changes in gut microbial populations. Our observations are consistent with previous in vitro experiments using porcine cecal contents to create an in vitro model of gut microbial metabolism which showed marked decrease of fecal 


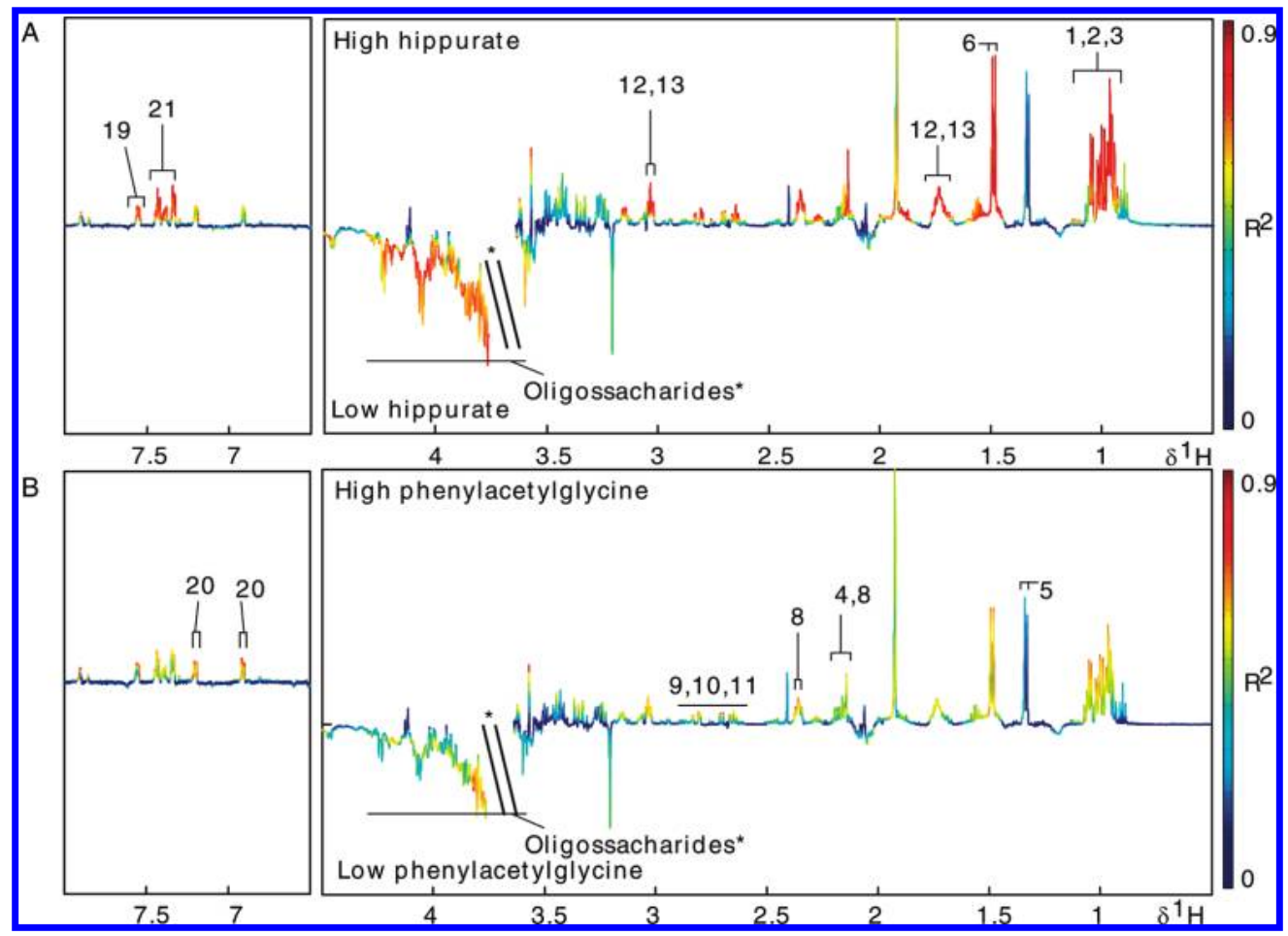

Figure 5. O-PLS regression of (A) urinary hippurate and (B) urinary phenylacetylglycine against the fecal extract ${ }^{1} \mathrm{H}$ NMR spectra profiles. See Figures 1 and 2 for metabolite key.

Table 3. O-PLS Model Summary of the Correlations Between Urinary Hippurate and Phenylacetylglycine with Fecal Metabolites for Day $1\left(D_{1}\right)$, Day $3\left(D_{3}\right)$, Day $7\left(D_{7}\right)$, Day $13\left(D_{13}\right)$ and Day $19\left(D_{19}\right)$

\begin{tabular}{lcccccl}
\hline $\begin{array}{c}\text { time } \\
\text { point }\end{array}$ & $\begin{array}{c}\text { hippurate } \\
Q^{2}\end{array}$ & $R^{2}$ & $\begin{array}{c}\text { number of } \\
\text { components }\end{array}$ & $Q^{2}$ & $R^{2}$ & $\begin{array}{c}\text { PAmber of } \\
\text { components }\end{array}$ \\
\hline $\mathrm{D}_{1}$ & $71 \%$ & $57 \%$ & 1 & $<0$ & - & - \\
$\mathrm{D}_{3}$ & $56 \%$ & $64 \%$ & 3 & $37 \%$ & $60 \%$ & 2 \\
$\mathrm{D}_{7}$ & $63 \%$ & $64 \%$ & 5 & $13 \%$ & $20 \%$ & 1 \\
$\mathrm{D}_{13}$ & $52 \%$ & $38 \%$ & 3 & $<0$ & - & - \\
$\mathrm{D}_{19}$ & $17 \%$ & $22 \%$ & 1 & $<0$ & - & -
\end{tabular}

SCFAs following vancomycin intervention. ${ }^{47}$ In particular, fermentation of carbohydrates by the Bifidobacteria normally induces a decrease in the $\mathrm{pH}$ of the cecal content associated quantitatively with different SCFA profiles of treated animals as compared to control animals, which inhibits the growth of pathogens. ${ }^{45,48}$ The disruption of the microbial production of SCFAs after vancomycin administration might thus be expected to profoundly disturb the gut microbial environment and alter relative amounts, and composition of colonic bacterial species. $^{48}$ Given that most of the SCFAs are rapidly absorbed in the ascending part of the colon and represent potential substrates for the liver and various tissues, such antibioticinduced effects might have significant nutritional consequences for the host. ${ }^{45,49,50}$ The disruption of the SCFA bacterial metabolism results in a loss of bioavailability of these metabolites for the host metabolism, possibly related to the observed reduction in urinary elimination of $n$-butyrate and $\alpha$-ketoisovalerate (Table 2). In addition, the reduction in $n$-butyrate production might have physiological consequences for individual animals because bacterial $n$-butyrate has a 'trophic' effect on colonocytes. ${ }^{2,45}$ Previous studies have shown the contribution of gut microbiota to mammalian host metabolism by measuring specific groups of compounds such as amino acids, bile acids, SCFAs, monitoring changes in fecal ammonia and host physiological descriptors such as those related to energy expenditure, lipoprotein profile and recovery from gut dysbiosis. ${ }^{51-53}$ Interestingly, fecal lactate did not correlate with urinary hippurate (Figure 5A) and may reflect the fact that urinary lactate contains both the D- and L-enantiomers, of mixed host and microbial sources, whereas fecal lactate is predominantly produce by bacteria and therefore is mainly in the D-form.

Another prominent finding is the reduction in the concentrations of SCFAs including acetate and butyrate, together with increased concentrations of oligosaccharides in fecal water from vancomycin-treated mice compared with that from control mice on days $1,3,5$, and 7 . This is consistent with a previous study where Bender et al. ${ }^{47}$ employed an in vitro model for the semicontinuous incubation of defined colon contents to examine the effect of vancomycin on SCFAs composition. ${ }^{47}$ These SCFAs are derived from the fermentation of nongastric-digestible starches and oligosaccharides by saccharolytic bacteria. The composition of fermentation endproducts is dependent on the bacteria species, substrates and chemical environment, such as $\mathrm{pH} .{ }^{48}$ Rycroft et al. ${ }^{54}$ reported that galacto-oligosaccharides generated high levels of SCFAs and decreased the numbers of clostridia. ${ }^{54}$ Among these SCFAs, $n$-butyrate seems to be most beneficial to colon cellular functions, such as proliferation, membrane synthesis and sodium absorption, and it also shows effect of anti-colon carcinogenesis and anti-tumor activity. ${ }^{55,56}$ In the present study, the 'abnormal' levels of SCFAs, which lasted for 13 days, indicated a large amount of microbiota experienced reduced activities, unlike $C$. coccoides and $C$. leptum which recovered from day 2 onward. Interestingly, there was an increase in species diversity in the vancomycin-treated group supported by the increased number of DNA bands appearing on the DGGE. Although those species could not be unequivocally 
Table 4. Taxonomic Affiliation of the Partial $16 \mathrm{~S}$ rRNA Gene Sequence Obtained from the Excised DGGE Band ${ }^{a}$

\begin{tabular}{|c|c|c|c|c|c|c|c|}
\hline Phyla & Class & Order & Family & Genus & Species & $\begin{array}{c}\text { Accesion } \\
\text { number }\end{array}$ & Similarity (\%) \\
\hline Firmicutes & Mollicutes & Anaeroplasmatales & Anaeroplasmataceae & Anaeroplasma & $\begin{array}{l}\text { Uncultured } \\
\text { bacterium }\end{array}$ & AY960576 & 92 \\
\hline Firmicutes & Mollicutes & Anaeroplasmatales & Anaeroplasmataceae & Anaeroplasma & $\begin{array}{l}\text { Anaeroplasma } \\
\text { bactoclasticum }\end{array}$ & M25049 & 90 \\
\hline Firmicutes & Mollicutes & Anaeroplasmatales & Anaeroplasmataceae & Anaeroplasma & $\begin{array}{l}\text { Uncultured } \\
\text { bacterium }\end{array}$ & EU006469 & 92 \\
\hline Firmicutes & Clostridia & Clostridiales & Eubacteriaceae & Eubacterium & $\begin{array}{c}\text { Eubacterium } \\
\text { desmolans }\end{array}$ & L34618 & 83 \\
\hline Firmicutes & Clostridia & Clostridiales & Clostridiaceae & Clostridium & $\begin{array}{l}\text { Uncultured } \\
\text { bacterium }\end{array}$ & EF406679 & 97 \\
\hline Firmicutes & Clostridia & Clostridiales & Clostridiaceae & Clostridium & $\begin{array}{l}\text { Clostridium } \\
\text { cocleatum }\end{array}$ & AF028350 & 97 \\
\hline Firmicutes & Clostridia & Clostridiales & Clostridiaceae & Clostridium & $\begin{array}{l}\text { Uncultured } \\
\text { bacterium }\end{array}$ & EF603657 & 97 \\
\hline Firmicutes & Clostridia & Clostridiales & Clostridiaceae & Clostridium & $\underset{1}{\text { Clostridium sp. BA- }}$ & AB196728 & 83 \\
\hline Firmicutes & Clostridia & Clostridiales & Lachnospiraceae & Incertae sedis & $\begin{array}{l}\text { Uncultured } \\
\text { bacterium }\end{array}$ & EF704431 & 96 \\
\hline Firmicutes & Clostridia & Clostridiales & Clostridiaceae & Clostridium & $\begin{array}{l}\text { Clostridium } \\
\text { symbiosum }\end{array}$ & EF442669 & 95 \\
\hline Firmicutes & Clostridia & Clostridiales & Clostridiaceae & Ruminococcus & $\begin{array}{l}\text { Uncultured } \\
\text { bacterium }\end{array}$ & AY976513 & 58 \\
\hline Firmicutes & Clostridia & Clostridiales & Lachnospiraceae & Anaerostipes & $\begin{array}{c}\text { Butyrate-producing } \\
\text { bacterium SS2/1 }\end{array}$ & AY305319 & 60 \\
\hline Proteobacteria & Gammaproteobacteria & Enterobacteriales & Enterobacteriaceae & Escherichia & $\begin{array}{l}\text { Uncultured } \\
\text { bacterium }\end{array}$ & EF674507 & 94 \\
\hline Proteobacteria & Gammaproteobacteria & Enterobacteriales & Enterobacteriaceae & Photorhabdus & $\begin{array}{l}\text { Photorhabdus } \\
\text { luminescens }\end{array}$ & DQ518589 & 93 \\
\hline
\end{tabular}

${ }^{a}$ The rows shaded in gray are the closest hits to bacteria, which have been cultured.

identified here, this gross observation is in agreement with a previous human study, ${ }^{53}$ which reported a marked elevation in total numbers of Clostridium bacteria including C. bifermentans, C. clostridioforme, C. sordellii and C. malenominatum, and an increased species diversity in the elderly people under antibiotic treatment.

In the current study, lower fecal concentration of amino acid levels were observed in vancomycin-treated mice (Table 1, Figure 4), which may affect the complex fluxes of amino acids and protein nitrogen across the intestinal mucosa. ${ }^{57}$ Although gastrointestinal digestion of some dietary proteins is incomplete, only about $2-5 \%$ escapes small intestinal digestion/ absorption. Immense populations of bacteria living within the large intestine are key contributors to the digestion and metabolism of amino acids and vitamins in the gut. ${ }^{58}$ In particular, intestines in germ-free animals generally show changed gut morphology, including atypical epithelial structure, less efficient nutrient usage and intestinal inflammation. ${ }^{59-61}$ The antibiotic-induced disruption of the gut bacterial ecology may thus promote an inflammatory disruption of absorptive abilities of intestinal brush border, which could lead to malabsorption of amino acids and SCFAs. ${ }^{62}$ Additionally, since $<50 \%$ of the fecal mass generally consists of bacteria, ${ }^{2}$ it is conceivable that the amino acids extracted from feces might also be derived from the gut bacteria themselves and that the observed reduction in fecal amino acids reflects perturbed amino acids metabolism as well as reduced bacterial population due to vancomycin treatment.

Changes were noted in urinary $\alpha$-ketoisocaproate and creatine levels following antibiotic treatment. In the human gut, the anaerobic genus Clostridium were found to metabolize leucine to isocaproate and $\alpha$-ketoisocaproate; ${ }^{63}$ and creatine has also been shown to be co-metabolized in mammals and their gut microbiota. ${ }^{64,65}$ Perturbed gut microbial changes observed in SCFAs, oligosaccharides and amino acids indicated vancomycin-induced changes in metabolism of these compounds, which may also explain the change in the relative amounts of $\alpha$-ketoisocaproate and creatine in the urine.
This work has shown the potential of a metabolic profiling strategy for studying the effects of antibiotics on the gut microbiota and has provided insights into the metabolic changes associated with antibiotic treatment and its effect on gut microbiota modification. Furthermore, urinary hippurate levels correlated closely with the amino and SCFA profiles in the feces. Our metabolic profiling approach showed changes in host amino acids metabolism and SCFA metabolism as well as in host metabolism of phenolics due to antibiotic treatment. The methods used here were intended to look at general aspects of the gut microbiome and as such are not specific enough to determine which species are being altered and may be responsible for the changes in the host's metabolite profiles. Further studies using such methods as stable isotope probing, metabonomics and high-throughput sequencing of 16S rRNA genes are needed to start to determine the keystone species in the gut, which are responsible for generating the host's metabotype.

Abbreviations: COSY, ${ }^{1} \mathrm{H}-{ }^{1} \mathrm{H}$ correlation spectroscopy; FID, free induction decay; IBS, irritable bowel syndrome; NMR, nuclear magnetic resonance; O-PLS-DA, orthogonal signal correction-projection to latent structure-discriminant analysis; PCR-DGGE, polymerase chain reaction-denaturing gradient gel electrophoresis; RD, recycle delay; SCFA, short chain fatty acid; STOCSY, statistical total correlation spectroscopy; TMA, trimethylamine; TMAO, trimethylamine- $N$-oxide; TOCSY, ${ }^{1} \mathrm{H}-{ }^{1} \mathrm{H}$ total correlation spectroscopy; TSP, 3-trimethylsilyl-1-[2,2,3,3$\left.{ }^{2} \mathrm{H} 4\right]$ propionate.

Acknowledgment. We thank Prof. Reto Brun and Dr. Sonja Bernhard from the Swiss Tropical Institute for help with the animal experiment. This work received financial support from the International Study of Macro/micronutrients and Blood Pressure grant (1-R01-HL084228-01A1) to I.K.S.Y., Nestle for F.-P.M. and Y.W., the Swiss National Science Foundation (project no. PPOOB-102883, PPOOB119129) to J.V.L., J.S., and J.U., and Deputy Rector Award, Imperial College London to J.V.L. 


\section{References}

(1) Stephen, A. M.; Cummings, J. H. L. Med. Microbiol. 1980, 13, 4556.

(2) Guarner, F.; Malagelada, J.-R. Lancet 2003, 361, 512-519.

(3) Tannock, G. W. Int. J. Epidemiol. 2005, 34, 13-15.

(4) O'Hara, A. M.; Shanahan, F. EMBO Rep. 2006, 7, 688-693.

(5) Barbara, G.; Stanghellini, V.; Brandi, G.; Cremon, C.; Di Nardo, G.; De Giorgio, R.;.; Corinaldesi, R. Am. J. Gastroenterol. 2005, 100, 2560-2568.

(6) Thompson-Chagoyán, O. C.; Maldonado, J.; Gil, A. Dig. Dis. Sci. 2007, 52, 2069-2077.

(7) Sokol, H.; Seksik, P.; Rigottier-Gois, L.; Lay, C.; Lepage, P.; Podglajen, I.; Marteau, P.; Dore, J. Inflammatory Bowel Dis. 2006, 12, 106-111.

(8) Ley, R. E.; Turnbaugh, P. J.; Klein, S.; Gordon, J. I. Nature 2006, 444, 1023-1024.

(9) Turnbaugh, P. J.; Ley, R. E.; Mahowald, M. A.; Magrini, V.; Mardis, E. R.; Gordon, J. I. Nature 2006, 444, 1027-1031.

(10) Bäckhed, F.; Manchester, J. K.; Semenkovich, C. F.; Gordon, J. I. Proc. Natl. Acad. Sci. U.S.A. 2007, 104, 979-984.

(11) Phipps, A. N.; Stewart, J.; Wright, B.; Wilson, I. D. Xenobiotica 1998, $28,527-537$.

(12) Nicholls, A. W.; Mortishire-Smith, R. J.; Nicholson, J. K. Chem. Res. Toxicol. 2003, 16, 1395-1404

(13) Williams, R. E.; Eyton-Jones, H. W.; Farnworth, M. J.; Gallagher, R.; Provan, W. M. Xenobiotica 2002, 32, 783-794.

(14) Holmes, E.; Nicholson, J. K. Toxicol. Sci. 2005, 37, 1-2.

(15) Robosky, L. C.; Wells, D. F.; Egnash, L. A.; Manning, M. L.; Reily, M. D.; Robertson, D. G. Toxicol. Sci. 2005, 87, 277-284.

(16) Dumas, M. E.; Barton, R. H.; Toye, A.; Cloarec, O.; Blancher, C.; Rothwell, A.; Fearnside, J.; Tatoud, R.; Blanc, V.; Lindon, J. C.; Mitchell, S. C.; Holmes, E.; McCarthy, M. I.; Scott, J.; Gauguier, D.; Nicholson, J. K. Proc. Natl. Acad. Sci. U.S.A. 2006, 103, 1251112516.

(17) Martin, F.-P.; Dumas, M.-E.; Wang, Y.; Legido-Quigley, C.; Yap, I. K. S.; Tang, H.; Zirah, S.; Murphy, G. M.; Cloarec, O.; Lindon, J. C.; Sprenger, N.; Fay, L. B.; Kochhar, S.; van Bladeren, P.; Holmes, E.; Nicholson, J. K. Mol. Syst. Biol. 2007, 3, 112.

(18) Martin, F. P.; Verdu, E. F.; Wang, Y.; Dumas, M. E.; Yap, I. K. S.; Cloarec, O.; Bergonzelli, G. E.; Corthesy-Theulaz, I.; Kochhar, S.; Holmes, E.; Lindon, J. C.; Collins, S. M.; Nicholson, J. K. L. Proteome Res. 2006, 5, 2185-2193.

(19) Halligan, S.; Byard, S. J.; Spencer, A. J.; Gray, T. J.; Harpur, E. S.; Bonner, F. W. Toxicol. Lett. 1995, 81, 15-21.

(20) Murgatroyd, L. B.; Pickford, R. J.; Smith, I. K.; Wilson, I. D.; Middleton, B. L. Human Exp. Toxicol. 1992, 11, 35-41.

(21) Lenz, E. M.; Bright, J.; Knight, R.; Westwood, F. R.; Davies, D. Major, H.; Wilson, I. D. Biomarkers 2005, 10, 173-187.

(22) Ramo Rao, A. V.; Mukund, K.; Gurjar, K.; Reddy, L.; Srinivasa Rao, A. Chem. Rev. 1995, 95, 2135-2167.

(23) Pultz, N. J.; Stiefel, U.; Donskey, C. J. Antimicrob. Agents Chemother 2005, 49, 3513-3516.

(24) Nicholson, J. K.; Foxall, P. J.; Spraul, M.; Farrant, R. D.; Lindon, J. C. Anal. Chem. 1995, 67, 793-811.

(25) Fan, T. W. M. Prog. Nucl. Magn. Reson. Spectrosc. 1996, 28, 161219.

(26) Cloarec, O.; Dumas, M. E.; Craig, A.; Barton, R. H.; Trygg, J.; Hudson, J.; Blancher, C.; Gauguier, D.; Lindon, J. C.; Holmes, E.; Nicholson, J. K. Anal. Chem. 2005, 77, 1282-1289.

(27) Trygg, J. J. Chemom. 2002, 16, 283-293.

(28) Cloarec, O.; Dumas, M. E.; Trygg, J.; Craig, A.; Barton, R. H.; Lindon, J. C.; Nicholson, J. K.; Holmes, E. Anal. Chem. 2005, 77, 517-526.

(29) Vanhoutte, T.; Huys, G.; De Brandt, E.; Swings, J. FEMS Microbiol. Ecol. 2004, 48, 437-446.

(30) Scanlan, P. D.; Shanahan, F.; Clune, Y.; Collins, J. K.; O'Sullivan, G. C.; O'Riordan, M.; Holmes, E.; Wang, Y.; Marchesi, J. R. Environ. Microbiol. 2008, 10, 789-798.
(31) Wang, Q.; Garrity, G. M.; Tiedje, J. M.; Cole, J. R. Appl. Environ. Microbiol. 2007, 73, 5261-5267.

(32) De La Cochetière, M. F.; Durand, T.; Lepage, P.; Bourreille, A.; Galmiche, J. P.; Doré, J. J. Clin. Microbiol. 2005, 43, 5588-5592.

(33) Wilcox, M. H. Best Pract. Res. Clin. Gastroenterol. 2003, 17, 475493.

(34) Li, M.; Wang, B.; Zhang, M.; Rantalainen, M.; Wang, S.; Zhou, H.; Zhang, Y.; Shen, J.; Pang, X.; Zhang, M.; Wei, H.; Chen, Y.; Lu, H.; Zuo, J.; Su, M.; Qiu, Y.; Jia, W.; Xiao, C.; Smith, L. M.; Yang, S.; Holmes, E.; Tang, H.; Zhao, G.; Nicholson, J. K.; Li, L.; Zhao, L. Proc. Natl. Acad. Sci. U.S.A. 2008, 105, 2117-2122.

(35) Nicholson, J. K. Mol. Syst. Biol. 2006, 2, 52.

(36) Beckwith-Hall, B. M.; Holmes, E.; Lindon, J. C.; Gounarides, J.; Vickers, A.; Shapiro, M.; Nicholson, J. K. Chem. Res. Toxicol. 2002, $15,1136-1141$.

(37) Goodwin, B. L.; Ruthven, C. R.; Sandler, M. Biochem. Pharmacol. 1994, 47, 2294-2297.

(38) Schwab, A. J.; Tao, L.; Yoshimura, T.; Simard, A.; Barker, F.; Pang, K. S. Am. J. Physiol. Gastrointest. Liver Physiol. 2001, 280, G1124G1136.

(39) Asatoor, A. M. Biochim. Biophvs. Acta 1965, 100, 290-292.

(40) Diaz, E.; Ferrández, A.; Prieta, M. A.; Garcia, J. Microbiol. Mol. Biol. Rev. 2001, 65, 523-569.

(41) Zeisel, S. H.; Da Costa, K.; Fox, J. G. Biochem. J. 1985, 232, 403408.

(42) Smith, J. L.; Wishnok, J. S.; Deen, W. M. Toxicol. Appl. Pharmacol. 1994, 125, 296-308.

(43) Salanitro, J. P.; Wegener, W. S. J. Bacteriol. 1971, 108, 885-892.

(44) Titgemeyer, E. C.; Bourquin, L. D.; Fahey, G. C., Jr.; Garleb, K. A. Am. J. Clin. Nutr. 1991, 53, 1418-24.

(45) Topping, D. L.; Clifton, P. M. Physiol. Rev. 2001, 81, 1031-1064.

(46) Macfarlane, S.; Macfarlane, G. T. Proc. Nutr. Soc. 2007, 62, 67-72.

(47) Bender, A.; Breves, G.; Stein, J.; Leonhard-Marek, S.; Schroder, B.; Winckler, C. Z. Gastroenterol. 2001, 11, 911-918.

(48) Walker, A. W.; Duncan, S. H.; McWilliam Leith, E. C.; Child, M. W.; Flint, H. L. Appl. Environ. Microbiol. 2005, 71, 3692-3700.

(49) Wong, J. M.; de Souza, R.; Kendall, C. W.; Emam, A.; Jenkins, D. J. L. Clin. Gastroenterol. 2006, 40, 235-243.

(50) Oltmer, S.; von Engelhardt, W. Scand. J. Gastroenterol. 1994, 29, 1009-1016.

(51) Gonthier, M-P.; Verny, M-A.; Besson, C.; Remesy, C.; Scalbert, A. J. Nutr. 2003, 133, 1853-1859.

(52) Coldham, N. G.; Darby, C.; Hows, M.; King, L. J.; Zhang, A. Q.; Sauer, M. J. Xenobiotica 2002, 32, 45-62.

(53) Woodmansey, E. J.; McMurdo, M. E.; Macfarlane, G. T.; Macfarlane, S. Appl. Environ. Microbiol. 2004, 70, 6113-6122.

(54) Rycroft, C. E.; Jones, M. R.; Gibson, G. R.; Rastall, R. A. J. Appl. Microbiol. 2001, 91, 878-887.

(55) Zampa, A.; Silvi, S.; Fabiani, R.; Morozzi, G.; Orpianesi, C.; Cresci, A. Anaerobe 2004, 10, 19-26.

(56) Archer, S.; Meng, S. F.; Wu, J.; Johnson, J.; Tang, R.; Hodin, R. Surgerv 1998, 124, 248-253.

(57) Fuller, M. F.; Tome, D. J. AOAC Int. 2005, 88, 923-34.

(58) Cummings, J. H.; Macfarlane, G. T. L. Parenter. Enteral Nutr. 1997, $21,357-365$.

(59) Cebra, J. J. Am. J. Clin. Nutr. 1999, 69, 1046S-1051S.

(60) Gordon, J. I.; Hooper, L. V.; McNevin, M. S.; Wong, M.; Bry, L. Am. J. Physiol. 1997, 273, G565-570.

(61) Shanahan, F. Best Pract. Res. Clin. Gastroenterol. 2002, 16, 915931.

(62) Linskens, R. K.; Huijsdens, X. W.; Savelkoul, P. H.; VandenbrouckeGrauls, C. M.; Meuwissen, S. G. Scand. J. Gastroenterol. 2001, 36, 29-40.

(63) Kim, J.; Darley, D.; Selmer, T.; Buckel, W. Appl. Environ. Microbiol. 2006, 72, 6062-6069.

(64) Wyss, M.; Kaddurah-Daouk, R. Phvsiol. Rev. 2000, 80, 1107-1213.

(65) Brosnan, J. T.; Brosnan, M. E. Annu. Rev. Nutr. 2007, 27, 41-261.

\section{PR700864X}

